\title{
An asymptotic formula for the Chebyshev theta function
}

\section{Aditya Ghosh}

\author{
Indian Statistical Institute, Kolkata, India \\ e-mail: ghoshadi26@gmail.com
}

Received: 11 March 2019

Revised: 21 October 2019

Accepted: 23 October 2019

Abstract: Let $\left\{p_{n}\right\}_{n \geq 1}$ be the sequence of primes and $\vartheta(x)=\sum_{p \leq x} \log p$, where $p$ runs over the primes not exceeding $x$, be the Chebyshev $\vartheta$-function. In this note, we derive lower and upper bounds for $\vartheta\left(p_{n}\right) / n$, by comparing it with $\log p_{n+1}$ and deduce the asymptotic formula $\vartheta\left(p_{n}\right) / n=\log p_{n+1}\left(1-\frac{1}{\log n}+\frac{\log \log n}{\log ^{2} n}(1+o(1))\right)$.

Keywords: Chebyshev theta function, Geometric mean of first $n$ primes, Prime numbers.

2010 Mathematics Subject Classification: 11A41, 11A25.

\section{Introduction}

Let $\left\{p_{n}\right\}_{n \geq 1}$ be the sequence of the prime numbers and $\vartheta(x)=\sum_{p \leq x} \log p$, where $p$ runs over the primes not exceeding $x$, be the Chebyshev $\vartheta$-function. The type of bounds that we shall discuss here was introduced by Bonse [2], who showed that $\vartheta\left(p_{n}\right)>2 \log p_{n+1}$ holds for every $n \geq 4$ and $\vartheta\left(p_{n}\right)>3 \log p_{n+1}$ holds for every $n \geq 5$. Thereafter, Pósa [8] showed that, given any $k>1$, there exists $n_{k}$ such that $\vartheta\left(p_{n}\right)>k \log p_{n+1}$ holds for all $n \geq n_{k}$. Panaitopol [7] showed that in Pósa's result we can have $n_{k}=2 k$ and also gave the bound

$$
\frac{\vartheta\left(p_{n}\right)}{\log p_{n+1}}>n-\pi(n) \quad(n \geq 2)
$$

where $\pi(n)$ is equal to the number of primes less or equal to $n$. Hassani [5] refined Panaitopol's inequality to the following

$$
\frac{\vartheta\left(p_{n}\right)}{\log p_{n+1}}>n-\pi(n)\left(1-\frac{1}{\log n}\right) \quad(n \geq 101) .
$$


Recently, Axler [1, Propositions 4.1 and 4.5] showed that

$$
1+\frac{1}{\log p_{n}}+\frac{2.7}{\log ^{2} p_{n}}<\log p_{n}-\frac{\vartheta\left(p_{n}\right)}{n}<1+\frac{1}{\log p_{n}}+\frac{3.84}{\log ^{2} p_{n}},
$$

where the left-hand side inequality is valid for every integer $n \geq 218$ and the right-hand side inequality holds for every $n \geq 74004585$. This provides the following asymptotic formula

$$
\frac{\vartheta\left(p_{n}\right)}{n}=\log p_{n}-1-\frac{1}{\log p_{n}}+\Theta\left(\frac{1}{\log ^{2} p_{n}}\right) .
$$

For further terms, see Axler [1, Proposition 2.1].

In the present note, we show the following result, which is a refinement of (1).

Theorem 1.1. For all $n \geq 6$, we have

$$
n\left(1-\frac{1}{\log n}+\frac{\log \log n}{4 \log ^{2} n}\right) \leq \frac{\vartheta\left(p_{n}\right)}{\log p_{n+1}} \leq n\left(1-\frac{1}{\log n}+\frac{\log \log n}{\log ^{2} n}\right)
$$

The left-hand side inequality also holds for $2 \leq n \leq 6$.

We can, in fact, generalise the left-hand side of (2) to have the following result.

Theorem 1.2. For every $0<\varepsilon<1$, there exists $n_{\varepsilon} \in \mathbb{N}$ such that for every $n \geq n_{\varepsilon}$ it holds that

$$
n\left(1-\frac{1}{\log n}+(1-\varepsilon) \frac{\log \log n}{\log ^{2} n}\right) \leq \frac{\vartheta\left(p_{n}\right)}{\log p_{n+1}} \leq n\left(1-\frac{1}{\log n}+\frac{\log \log n}{\log ^{2} n}\right) .
$$

Corollary 1.2.1. We have $\frac{\vartheta\left(p_{n}\right)}{n}=\log p_{n+1}\left(1-\frac{1}{\log n}+\frac{\log \log n}{\log ^{2} n}(1+o(1))\right)$.

\section{Preliminaries}

Define $G(n, a)=\log n+\log \log n-1+\frac{\log \log n-a}{\log n}$. We shall use the following bounds for $\vartheta\left(p_{n}\right) / n$.

Lemma 2.1. For every $n \geq 3$, we have

$$
\frac{\vartheta\left(p_{n}\right)}{n} \geq G(n, 2.1454)
$$

and for every $n \geq 198$, we have

$$
\frac{\vartheta\left(p_{n}\right)}{n} \leq G(n, 2)
$$

Proof. The inequality (4) is due to Robin [9], and the inequality (5) was given by Massias and Robin [6]. 
Lemma 2.2. For every $n \geq 227$, we have

$$
p_{n} \leq n(\log n+\log \log n-0.8)
$$

and for every $n \geq 2$,

$$
p_{n} \geq n(\log n+\log \log n-1) .
$$

Proof. For $n \geq 8602$, we have the following stronger bound

$$
p_{n} \leq n(\log n+\log \log n-0.9385)
$$

given by Massias and Robin [6]. For $227 \leq n \leq 8601$ we verify the inequality (6) by direct computation. The inequality (7) is due to Dusart [4].

For the sake of brevity, we define $\mathcal{F}(n, \lambda)=1-\frac{1}{\log n}+\lambda \frac{\log \log n}{\log ^{2} n}$ and rewrite (2) as

$$
\mathcal{F}(n, 0.25) \log p_{n+1} \leq \vartheta\left(p_{n}\right) / n \leq \mathcal{F}(n, 1) \log p_{n+1}
$$

and rewrite (3) as

$$
\mathcal{F}(n, 1-\varepsilon) \log p_{n+1} \leq \vartheta\left(p_{n}\right) / n \leq \mathcal{F}(n, 1) \log p_{n+1}
$$

\section{Proof of Theorem 1.1}

The proof of Theorem 1.1 is split into two lemmas. In the first lemma, we give lower and upper bounds for $\log p_{n+1}$.

Lemma 3.1. For every $n \geq 140$, we have

$$
\log p_{n+1}<\log n+\log \log n+\frac{\log \log n-0.8+0.018}{\log n}=U(n)
$$

and for every $n \geq 2$, we have

$$
\log p_{n+1}>\log n+\log \log n+\frac{\log \log n-1}{\log n+0.5(\log \log n-1)}=V(n)
$$

Proof. First, we show that for every $x \geq 1$

$$
\frac{1}{x+0.4}>\log \left(1+\frac{1}{x}\right)>\frac{1}{x+0.5}
$$

In order to prove this, we set $f_{a}(x)=\log (1+x)-\frac{x}{1+a x}$. Note that, $f_{a}^{\prime}(x)=\frac{x\left(a^{2} x+2 a-1\right)}{(1+x)(1+a x)^{2}}$. Hence, $f_{0.4}^{\prime}(x)<0$ for every $x \in(0,1.25)$ which yields $f_{0.4}(1 / x)<f_{0.4}(0)=0$ for every $x \geq 1$. 
On the other hand, $f_{0.5}^{\prime}(x)>0$ for all positive $x$, which gives $f_{0.5}(1 / x)>f_{0.5}(0)=0$ for every $x \geq 1$.

Next, we give a proof of (11). By (6), we have for $n \geq 227$,

$$
\log p_{n+1} \leq \log (n+1)+\log (\log (n+1)+\log \log (n+1)-0.8) .
$$

The left-hand side inequality of (13) gives $\log (n+1)<\log n+\frac{1}{n+0.4}$, which in turn implies that

$$
\log \log (n+1)<\log \log n+\log \left(1+\frac{1}{(n+0.4) \log n}\right)<\log \log n+\frac{1}{(n+0.4) \log n} .
$$

Applying this to (14), we obtain for $n \geq 227$,

$$
\begin{aligned}
\log p_{n+1} & <\log n+\frac{1}{n+0.4}+\log \left(\log n+\log \log n-0.8+\frac{1+1 / \log n}{n+0.4}\right) \\
& <\log n+\log \log n+\frac{\log \log n-0.8}{\log n}+\frac{1}{\log n} \cdot \frac{\log n+1+1 / \log n}{n+0.4} .
\end{aligned}
$$

Now, $g(x)=\frac{\log x+1+1 / \log x}{x+0.4}$ is a decreasing function for $x \geq 2$ with $g\left(e^{5.99}\right) \leq 0.018$. Hence $g(x) \leq 0.018$ holds for every $x \geq 400>e^{5.99}$. Combined with (15), it yields that $\log p_{n+1}<U(n)$ for every $n \geq 400$. For every $140 \leq n \leq 399$ we check the inequality (11) with a computer. This completes the proof of (11).

To prove the inequality (12), first note that (7) gives for every $n \geq 1$,

$$
\log p_{n+1} \geq \log (n+1)+\log (\log (n+1)+\log \log (n+1)-1) .
$$

The right-side inequality of (13) gives $\log (n+1)>\log n+\frac{1}{n+0.5}$. Using (13) once again, we get, for $n \geq 2$,

$$
\log \log (n+1)-\log \log n>\log \left(1+\frac{1}{(n+0.5) \log n}\right)>\frac{1}{(n+0.5) \log n+0.5} .
$$

Applying this to (16), we arrive at

$$
\begin{aligned}
\log p_{n+1} & >\log n+\log \left(\log n+\frac{1}{n+0.5}+\log \log n+\frac{1}{(n+0.5) \log n+0.5}-1\right) \\
& >\log n+\log \log n+\log \left(1+\frac{\log \log n-1}{\log n}\right) .
\end{aligned}
$$

Invoking (13) one more time, we get $\log p_{n+1}>V(n)$ for every $n \geq 2$.

Lemma 3.2. For every $n \geq 396$, we have

$$
G(n, 2.1454) \geq \mathcal{F}(n, 0.25) \cdot U(n),
$$

and for every $n \geq 2$, we have

$$
G(n, 2) \leq \mathcal{F}(n, 1) \cdot V(n) .
$$

Here $U(n)$ and $V(n)$ are defined as in Lemma 3.1. 
Proof. We start with the proof of (17). Setting $x=\log n$, the inequality (17) can be rewritten as

$$
x+\log x-1+\frac{\log x-2.1454}{x} \geq\left(1-\frac{1}{x}+\frac{\log x}{4 x^{2}}\right)\left(x+\log x+\frac{\log x-0.8+0.018}{x}\right),
$$

which is equivalent to

$$
\left(\frac{3}{4} \log x+\frac{\log x}{x}\right)+\left(-2.1454-\frac{\log ^{2} x}{4 x}-\frac{\log ^{2} x}{4 x^{2}}\right)+(0.8-0.018)\left(1-\frac{1}{x}+\frac{\log x}{4 x^{2}}\right) \geq 0 .
$$

The left-hand side is a sum of three increasing functions on the interval $[5.7, \infty)$ and at $x=5.99$ the left-hand side is positive. So the last inequality holds for every $x \geq 5.99$; i.e., for every $n \geq 400$. A direct computation shows that the inequality (17) also holds for every $n$ satisfying $396 \leq n \leq 399$.

Next, we give a proof of (18). It is easy to see that

$$
x^{2}+\log x(\log x-1)>\frac{x}{2} \log x(\log x-1)
$$

for every $x>0$. Now, for $x \geq 1$, the last inequality is seen to be equivalent to

$$
\left(1-\frac{1}{x}+\frac{\log x}{x^{2}}\right) \frac{\log x-1}{x+0.5(\log x-1)} \geq \frac{\log x-2}{x} .
$$

Since $\frac{\log ^{2} x}{x^{2}} \geq 0$ for every $x>0$, we get

$$
\frac{\log ^{2} x}{x^{2}}+\left(1-\frac{1}{x}+\frac{\log x}{x^{2}}\right) \frac{\log x-1}{x+0.5(\log x-1)} \geq \frac{\log x-2}{x}
$$

for every $x \geq 1$. Substituting $x=\log n$ in (19), we obtain the inequality (18) for every integer $n \geq 3$. We can directly check that (18) holds for $n=2$ as well.

Finally, we give a proof of Theorem 1.1.

Proof of Theorem 1.1. We use (4), (17) and (11) respectively to see that for every $n \geq 396$,

$$
\vartheta\left(p_{n}\right) / n \geq G(n, 2.1454) \geq \mathcal{F}(n, 0.25) U(n)>\mathcal{F}(n, 0.25) \log p_{n+1} .
$$

A direct computation shows that the left-hand side inequality of (9) also holds for every integer $n$ with $2 \leq n \leq 395$.

In order to prove the right-hand side inequality of (9), we combine (5), (18) and (12), respectively, to get

$$
\vartheta\left(p_{n}\right) / n \leq G(n, 2) \leq \mathcal{F}(n, 1) V(n) \leq \mathcal{F}(n, 1) \log p_{n+1}
$$

for every $n \geq 198$. For smaller values of $n$, we use a computer. 


\section{Proof of Theorem 1.2}

The right-hand side of (10) has been established already. To show the left-hand side, we start with the following lemma.

Lemma 4.1. For any $0<\varepsilon<1$, there exists $m_{\varepsilon} \in \mathbb{N}$ such that

$$
G(n, 2.1454) \geq \mathcal{F}(n, 1-\varepsilon) \cdot U(n)
$$

holds for every $n \geq m_{\varepsilon}$. Here $U(n)$ is defined as in Lemma 3.1.

Proof. Fix any $0<\varepsilon<1$. We denote $a=2.1454, b=0.8-0.018$ and $\operatorname{set} x=\log n$ to transform the inequality (20) into

$$
x+\log x-1+\frac{\log x-a}{x} \geq\left(1-\frac{1}{x}+(1-\varepsilon) \frac{\log x}{4 x^{2}}\right)\left(x+\log x+\frac{\log x-b}{x}\right) .
$$

This is equivalent to

$$
\left(\varepsilon \log x+\frac{\log x}{x}\right)+\left(-a-(1-\varepsilon)\left(\frac{\log ^{2} x}{x}+\frac{\log ^{2} x}{x^{2}}\right)\right)+b\left(1-\frac{1}{x}+(1-\varepsilon) \frac{\log x}{x^{2}}\right) \geq 0 .
$$

Now, the left-hand side is a sum of three functions, each of which is strictly increasing for all sufficiently large $x$, and the limit of the left-hand side, as $x \rightarrow \infty$, is $+\infty$. Therefore we conclude that the last inequality holds for all sufficiently large $x$.

Proof of Theorem 1.2. For any $0<\varepsilon<1$, we have $m_{\varepsilon} \in \mathbb{N}$ such that (20) holds for every $n \geq m_{\varepsilon}$. We combine this with (4) and (11) to obtain that for every $n \geq n_{\varepsilon}:=\max \left\{m_{\varepsilon}, 140\right\}$

$$
\vartheta\left(p_{n}\right) / n \geq G(n, 2.1454) \geq \mathcal{F}(n, 1-\varepsilon) U(n) \geq \mathcal{F}(n, 1-\varepsilon) \log p_{n+1} .
$$

This completes the proof.

\section{Remarks}

1. For every $n \geq 599$, we have

$$
\frac{\pi(n)}{n} \geq \frac{1}{\log n}+\frac{1}{\log ^{2} n}
$$

which was found by Dusart [3]. Using this and a computer, we get

$$
\frac{\pi(n)}{n} \geq \frac{1}{\log n-1}\left(1-\frac{\log \log n}{4 \log n}\right)
$$

for every integer $n \geq 83$. Hence, (2) is an improvement of (1).

2. The bounds given in (2) are particularly useful for comparing $\vartheta\left(p_{n}\right) / n$ with $\log p_{n+1}$. To see a numerical example, we use a computer to find that for $n \geq 23$ the relative error in approximating $\vartheta\left(p_{n}\right) / n$ with $\mathcal{F}(n, 0.25)$ is less than $5 \%$ and for $n \geq 114$ it is less than $2 \%$. An important feature of (2) is that it holds even for very small values of $n$. 


\section{Acknowledgements}

I am thankful to Mridul Nandi (Indian Statistical Institute, Kolkata, India) and Mehdi Hassani (University of Zanjan, Iran) for their valuable suggestions.

\section{References}

[1] Axler, C. (2018). On the arithmetic and geometric means of the first $n$ prime numbers, Mediterr. J. Math., 15 (3), Art. 93, 21 pages.

[2] Bonse, H. (1907). Über eine bekannte Eigenschaft der Zahl 30 und ihre Verallgemeinerung, Archiv Math. Phys., 3 (12), 292-295.

[3] Dusart, P. (1998). Autour de la fonction qui compte le nombre de nombres premiers, $\mathrm{PhD}$ Thesis, Limoges.

[4] Dusart, P. (1999). The $k$-th prime is greater than $k(\ln k+\ln \ln k-1)$ for $k \geq 2$, Math. Comp., 68 (225), 411-415.

[5] Hassani, M. (2005). Approximation of the product $p_{1} p_{2} \cdots p_{n}$, RGMIA Research Report Collection, 8 (2), Article 20.

[6] Massias, J.-P., \& Robin, G. (1996). Bornes effectives pour certaines fonctions concernant les nombres premiers, J. Théor. Nombres de Bordeaux, 8, 213-238.

[7] Panaitopol, L. (2000). An inequality involving prime numbers, Univ. Beograd. Publ. Elektrotehn. Fak. Ser. Mat., 11, 33-35.

[8] Pósa, L. (1960). Über eine Eigenschaft der Primzahlen, Mat. Lapok, 11, 124-129.

[9] Robin, G. (1983). Estimation de la fonction de Tchebychef $\theta$ sur le $k$-ième nombre premier et grandes valeurs de la fonction $\omega(n)$ nombre de diviseurs premiers de $n$, Acta Arith., 42 (4), 367-389. 